\title{
Microscopic Image Retrieval Scheme Using Neural Network For Multi Image Queries
}

\author{
Jaipal Reddy Yeruva a, Dr. Anil Kumar b \\ ${ }^{a}$ Research Scholar, Dept. of Electronics and Communication Engineering, \\ Sri Satya Sai University of Technology \& Medical Sciences, Sehore, Bhopal Indore Road, Madhya Pradesh, India \\ ${ }^{\mathbf{b}}$ Research Guide, Dept. of Electronics and Communication Engineering, \\ Sri Satya Sai University of Technology \& Medical Sciences, Sehore, Bhopal Indore Road, Madhya Pradesh, India
}

Article History: Received: 11 January 2021; Accepted: 27 February 2021; Published online: 5 April 2021

\begin{abstract}
In this paper, we describe the plan and advancement of a neural network based image retrieval framework for microscopic images using a reference information base that contains images of more than one information. Such an extraction requires a point by point assessment of retrieval execution of image highlights. This paper presents a survey of crucial parts of content based image retrieval including highlight extraction of color and surface highlights. The proposed neural network based image retrieval framework utilizes a multitier way to deal with arrange and recover microscopic images including their particular subtypes, which are generally hard to separate and characterize. Broad examinations on neural network based image retrieval frameworks show that low-level image highlights can't generally depict elevated level semantic ideas in the clients mind. This framework empowers multi-image inquiry to ensure the semantic consistency among the recovered images. New weighting terms, roused from information retrieval hypothesis, are characterized for multiple-image inquiry and retrieval. The multi-image inquiry calculation with the proposed weighting technique accomplishes about normal order exactness at the main position retrieval, beating the image-level retrieval precision by about ideal rate focuses for different infections separately. Utilizing low level highlights just does exclude human insight. In the event that human mediation is permitted in the image retrieval framework the proficiency supports up.
\end{abstract}

Keywords: Neural Network, Microscopic Image, Retrieval, Multi Image, Queries.

\section{Introduction}

In Neural Network the neurons in a single layer don't interface with all the neurons in the following layer. Or maybe, a convolutional neural network utilizes a three-dimensional structure, where each set of neurons dissects a particular locale or "highlight" of the image. CNNs channels associations by nearness (pixels are just examined comparable to pixels close by), making the training cycle computationally attainable. In a CNN each gathering of neurons centers around one piece of the image. For instance, in a feline image, one gathering of neurons may distinguish the head, another the body, another the tail, and so forth There might be a few phases of division wherein the neural network image acknowledgment calculation investigates more modest pieces of the images, for instance, inside the head, the feline's nose, stubbles, ears, and so on The last output is a vector of probabilities, which predicts, for each element in the image, that it is so prone to have a place with a class or classification.

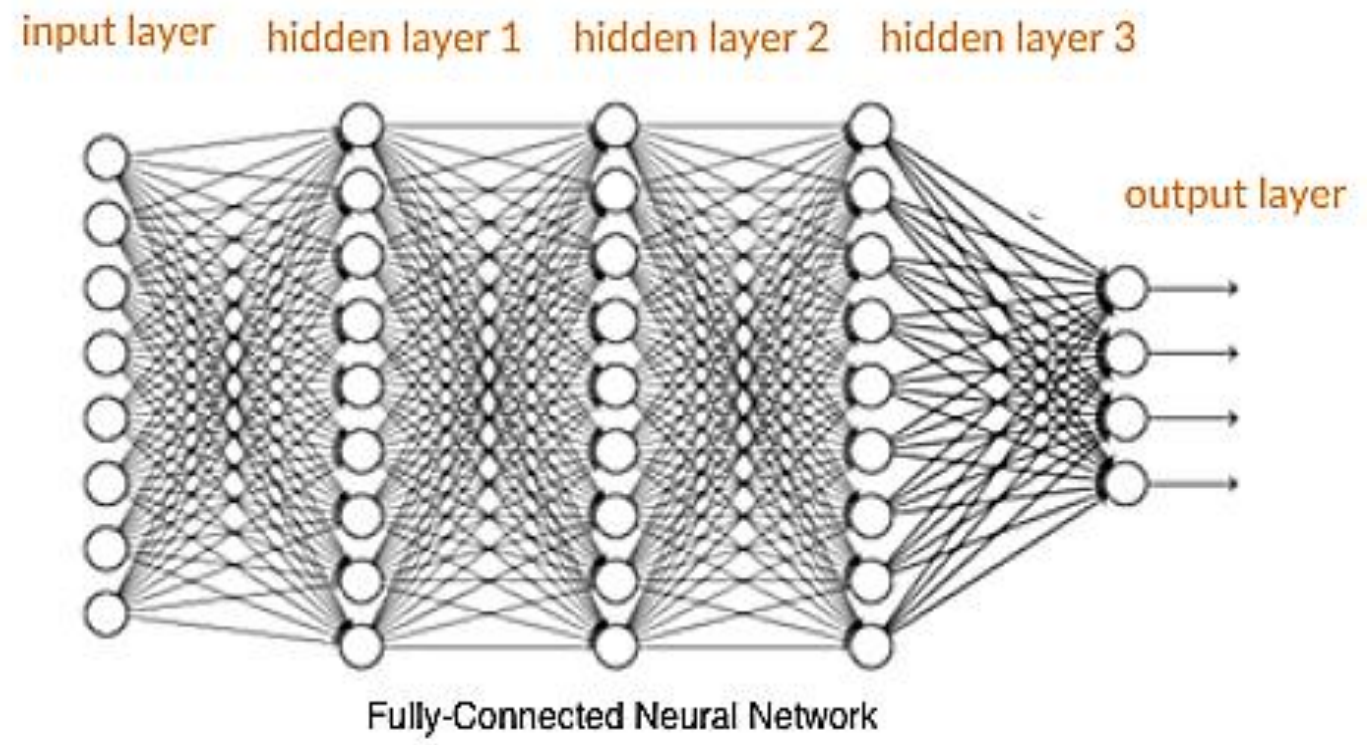

Figure 1 Neural Network

When training images are readied, you'll need a framework that can cycle them and use them to make a forecast on new, obscure images. That framework is a fake neural network. Neural network image acknowledgment calculations can arrange pretty much anything, from text to images, sound records, and recordings (see our top to 
bottom article on characterization and neural networks). Neural networks are an interconnected assortment of hubs called neurons or perceptron's. Each neuron takes one bit of the input information, ordinarily one pixel of the image, and applies a straightforward calculation, called an actuation capacity to produce an outcome. Every neuron has a mathematical weight that influences its outcome. That outcome is taken care of to extra neural layers until toward the finish of the cycle the neural network creates a forecast for each input or pixel. A CNN design makes it conceivable to foresee items and countenances in images utilizing industry benchmark datasets with up to $95 \%$ precision, more prominent than human abilities which remain at $94 \%$ exactness. All things considered, convolutional neural networks have their constraints: Require high handling power. Models are commonly trained on significant expense machines with particular Graphical Processing Units (GPUs). Can bomb when images are pivoted or inclined, or when an image has the highlights of the ideal item, yet not in the right request or position, for instance, a face with the nose and mouth exchanged around. Another design called CAPS Net has arisen to address this constraint.
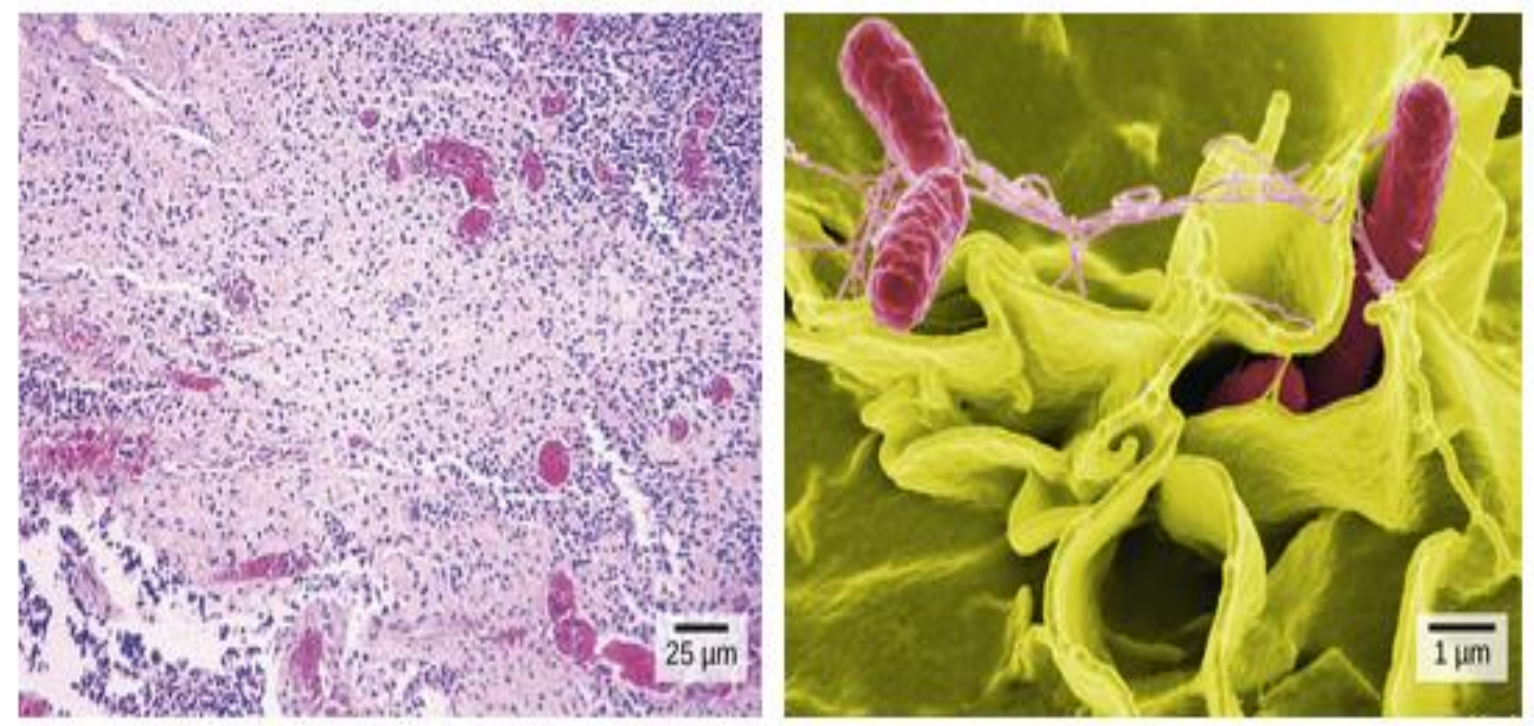

Figure 2 Microscopic Images

Despite the fact that cells fluctuate in size, they're for the most part minuscule. For example, the width of a commonplace human red platelet is around eight micrometers (0.008 millimeters). To give you some unique circumstance, the top of a pin is around one millimeter in width, so around 125 red platelets could be arranged in succession across the top of a pin. With a couple of exemptions, singular cells can't be seen with the unaided eye, so researchers must rather utilize magnifying instruments (miniature = "little"; - scope = "to take a gander at") to consider them. A magnifying lens is an instrument that amplifies protests in any case too little to even consider being seen, creating an image wherein the article seems bigger. Most photos of cells are taken utilizing a magnifying lens, and these photos can likewise be called micrographs. From the definition above, it may seem like a magnifying instrument is only a sort of amplifying glass. Indeed, amplifying glasses do qualify as magnifying instruments; since they have only one focal point, they are called basic magnifying lens. The fancier instruments that we ordinarily consider as magnifying lens are compound magnifying lens, implying that they have multiple focal points. On account of the manner in which these focal points are orchestrated, they can twist light to create a significantly more amplified image than that of an amplifying glass. In a compound magnifying instrument with two focal points, the plan of the focal points has an intriguing result: the direction of the image you see is flipped according to the genuine item you're inspecting. For instance, on the off chance that you were taking a gander at a bit of newsprint with the letter "e" on it, the image you saw through the magnifying instrument would be "ə." $\{1\}$ start superscript, 1, end superscript More perplexing compound magnifying instruments may not create a transformed image since they incorporate an extra focal point that "re-modifies" the image back to its ordinary state.

\section{LITERATURE REVIEW}

Kashif (2020): The fundamental issue in content-based image retrieval (CBIR) frameworks is the semantic hole which should be decreased for effective retrieval. The basic imaging signs which show up in the patient's lung CT filter assume a huge job in the distinguishing proof of harmful lung knobs and numerous other lung illnesses. In this paper, we propose another mix of descriptors for the powerful retrieval of these imaging signs. To begin with, we develop an element information base by joining neighborhood ternary example, nearby stage 
quantization, and discrete wavelet change. Next, joint shared information based component determination is conveyed to decrease the repetition and to choose an ideal list of capabilities for CISs retrieval. To this end, closeness estimation is performed by consolidating visual and semantic information in equivalent extent to develop a reasonable diagram and the shortest way is figured for learning logical likeness to get last comparability between each inquiry and data set image. The proposed framework is assessed on an openly available information base of lung CT imaging signs, and results are recovered based on visual component closeness examination and diagram based comparability correlation. The proposed framework accomplishes a mean normal accuracy (MAP) of $60 \%$ and 0.48 AUC of exactness review) chart utilizing just visual highlights likeness correlation. These outcomes further enhance chart based likeness measure with a MAP of $70 \%$ and 0.58 AUC which shows the predominance of our proposed conspire.

Abeer Al-Mohamade (2020): We propose a novel multiple question retrieval approach, named weight-student, which depends on visual component separation to estimate the distances between the inquiry images constantly in the information base. For each inquiry image, this segregation comprises of learning, in an unaided way, the ideal pertinence weight for each visual element/descriptor. These component pertinence loads are intended to lessen the semantic hole between the extricated visual highlights and the client's significant level semantics. We mathematically detail the proposed arrangement through the minimization of some goal capacities. This streamlining intends to create ideal element significance loads as for the client question. The proposed approach is surveyed utilizing an image assortment from the Corel information base. The expansion of interpersonal organizations alongside the wide scattering of keen gadgets has yielded a dramatic development of advanced image information bases. This gigantic increment has encouraged the test of mining explicit images among enormous assortments. Subsequently, image retrieval has become a functioning field of examination. Text-based image retrieval frameworks require the comment of images in an information base. This is a dull and costly assignment. Content-based image retrieval (CBIR) speaks to an elective that defeats this disadvantage. Thusly, the client chooses an inquiry image that passes on the information the person in question is searching for. The inquiry content is then used to mine the information base. A few CBIR approaches have been accounted for in the writing.

A.I. Shahin (2019): Microscopic images settling on choice issues include inaccuracy, vulnerability, dubiousness, irregularity, deficiency, and indeterminacy. Such impediments are because of the presence of multi objects, object direction, multi appearance colors, and changed staining degrees. Subsequently, NSs are assuming a huge part in taking care of such issues. NSs contributed in expanding the exhibition of a few MIA frameworks on various tissues from the human body, including upgrade of blood smear images, division of WBCs inside blood smear microscopic images, division of the glomerular cellar film from kidney tissue, lastly mitosis identification from bosom tissue. Subsequently, this part featured the significance of NSs in various microscopic applications in the MIA area to assist specialists with NS applications. The first investigations set up the basic job of NS in the MIA applications. Moreover, the NSs similitude estimation gives more information about NS. The comparability estimation calculation under multi measures has been utilized with fixed loads coefficients and versatile loads coefficients. On clinical image preparing, for example, CT, MRI, and ultrasound, the handled image is typically a grayscale power image. On the microscopic images case, these images are available in the RGB color format. The NSs have prepared diverse color space segments, which can be useful with other microscopic tissue images.

\section{PROPOSED METHODOLOGY}

This paper tells the best way to train a neural network, at that point utilize a neural network to estimate a highgoal image from a solitary low-goal image. The model tells the best way to train a NEURAL network and furthermore gives a pre-trained neural network. On the off chance that you decide to train the neural network, utilization of a CUDA-able NVIDIA GPU with register capacity 3.0 or higher is enthusiastically suggested. 


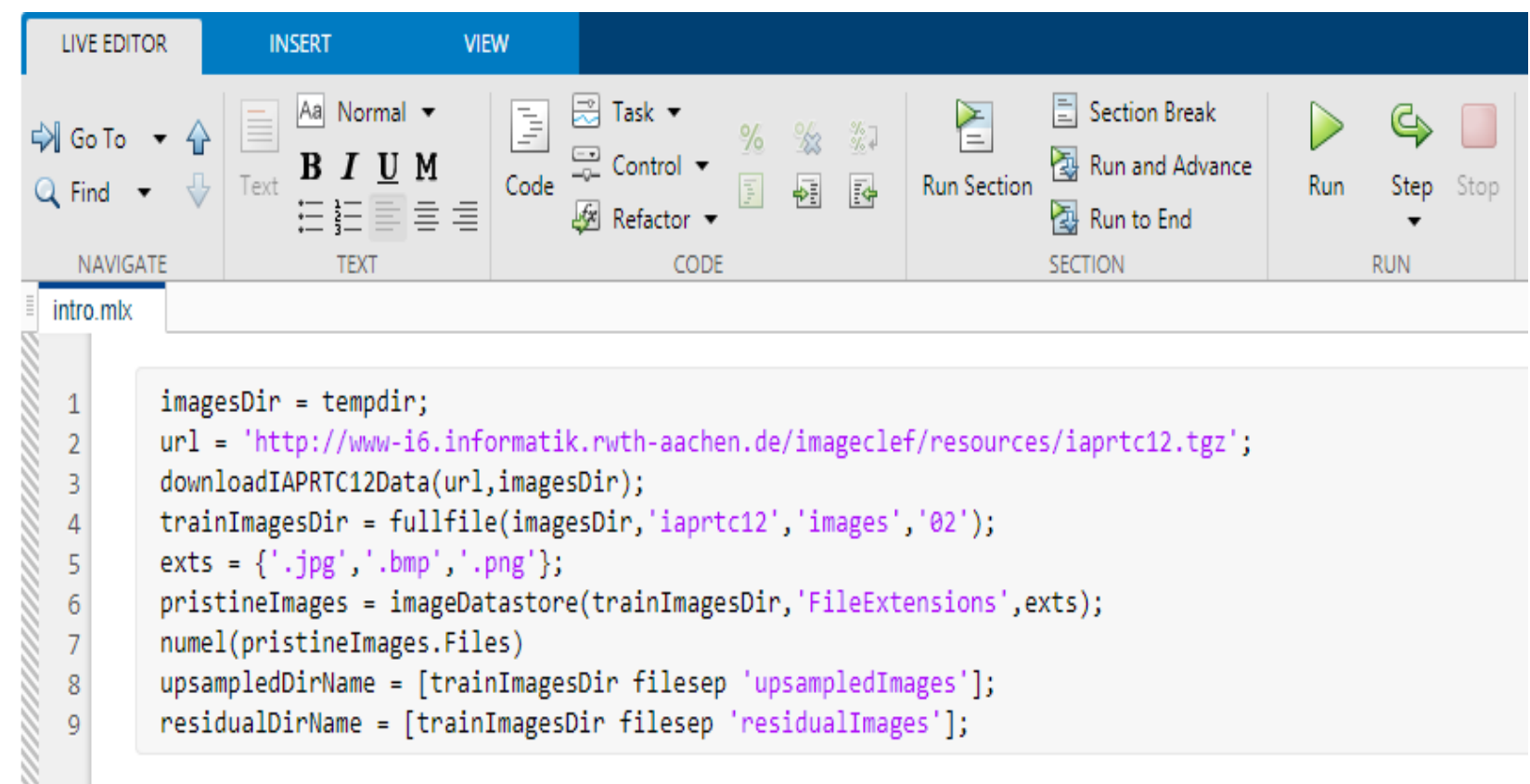

Figure 3Neural Network Image Training

Utilization of a GPU requires the Parallel Computing Toolbox. Multi image question is the way toward making high-goal images from low-goal images. Download the IAPR TC-12 Benchmark, which comprises of 20,000 still regular images. The informational index incorporates photographs of individuals, creatures, urban areas, and then some. The size of the information record is $\sim 1.8 \mathrm{~GB}$. In the event that you would prefer not to download the training informational index, at that point you can stack the pre-trained neural network by composing load ('trainedNEURAL-Epoch-100-ScaleFactors-234.mat'); at the order line. At that point, go straightforwardly to the Perform Single Image Super-Resolution Using neural network. Utilize the partner work, downloadIAPRTC12Data, to download the information.

This execution considers single image super-goal, where the objective is to recuperate one high-goal image from one low-goal image. It is testing since high-recurrence image content regularly can't be recuperated from the low-goal image. Without high-recurrence information, the nature of the high-goal image is restricted. Further, Image goal is a poorly presented issue since one low-goal image can yield a few potential high-goal images. It a convolutional neural network engineering intended to perform single image super-goal. The neural network learns the planning among low-and high-goal images. This planning is conceivable in light of the fact that lowgoal and high-goal images have comparable image content and contrast principally in high-recurrence subtleties. Neural network utilizes a lingering learning methodology, implying that the network figures out how to estimate a leftover image.

With regards to super-goal, a lingering image is the contrast between a high-goal reference image and a low-goal image that has been upscale utilizing bi-cubic interjection to match the size of the reference image. A remaining image contains information about the high-recurrence subtleties of an image. The neural network recognizes the remaining image from the luminance of a color image. The luminance channel of an image, Y, speaks to the brilliance of every pixel through a straight blend of the red, green, and blue pixel esteems. Interestingly, the two chrominance channels of an image, $\mathrm{Cb}$ and $\mathrm{Cr}$, are distinctive direct mixes of the red, green, and blue pixel esteems that speak to color-contrast information. Neural network is trained utilizing just the luminance channel since human insight is more delicate to changes in brilliance than to changes in color. In the event that Y higher is the luminance of the high-goal image and $\mathrm{Y}$ brings down is the luminance a low-goal image that has been upscale utilizing bi-cubic interjection, at that point the input to the NEURAL network is $\mathrm{Y}$ brings and the network learns down to anticipate $\mathrm{Y}$ remaining $=\mathrm{Y}$ higher $-\mathrm{Y}$ brings down from the training information. After the neural network figures out how to estimate the leftover image, you can remake high-goal images by adding the estimated lingering image to the up examined low-goal image, at that point changing over the image back to the RGB color space. 


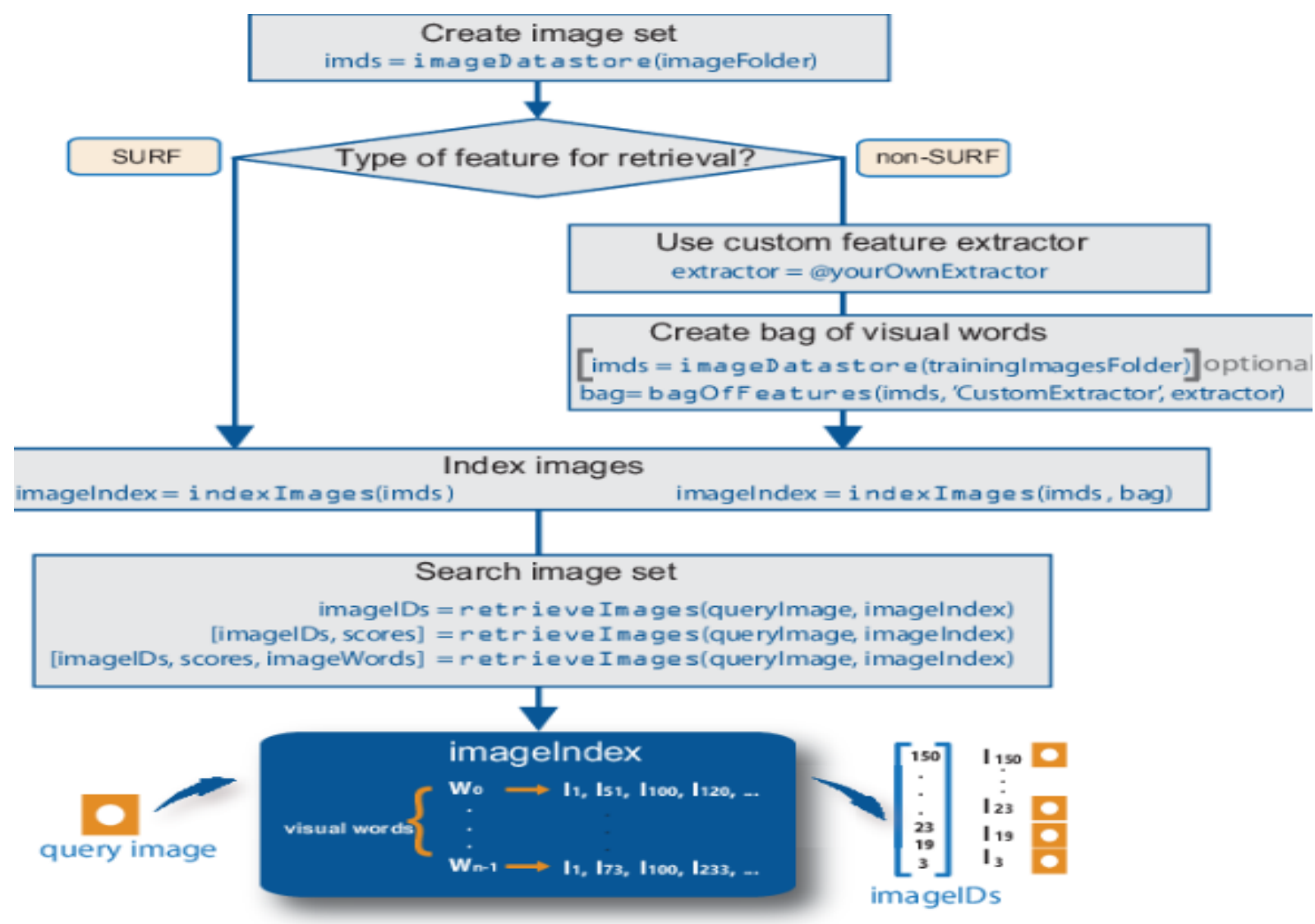

Figure 4 Image Retrieval Process

A scale factor relates the size of the reference image to the size of the low-goal image. As the scale factor builds, SISR turns out to be all the more badly presented on the grounds that the low-goal image loses more information about the high-recurrence image content. Neural takes care of this issue by utilizing a huge responsive field. This model trains a neural network with multiple scale factors utilizing scale expansion. Scale growth improves the outcomes at bigger scope factors on the grounds that the network can exploit the image setting from more limited size factors. Also, the neural network can sum up to acknowledge images with no number scale factors.

\section{EXPERIMENT RESULT}

In this part we going to reproduce and print consequence of microscopic image retrieval utilizing neural network. The input image is given to framework for information retrieval. In the image information base frameworks geological guides, pictures, clinical images, pictures in clinical map books, pictures acquiring by cameras, magnifying instruments, telescopes, camcorders, works of art, drawings and structures plans, drawings of mechanical parts, space images are considered as images. There are various models for color image portrayal. Thusly, he previously comprehended that white light is made out of numerous colors. Commonly, the PC screen can show $2^{\wedge} 8$ or 256 unique shades of dim. For color images this makes $2^{\wedge}(3 \times 8)=16,777,216$ distinct colors. 


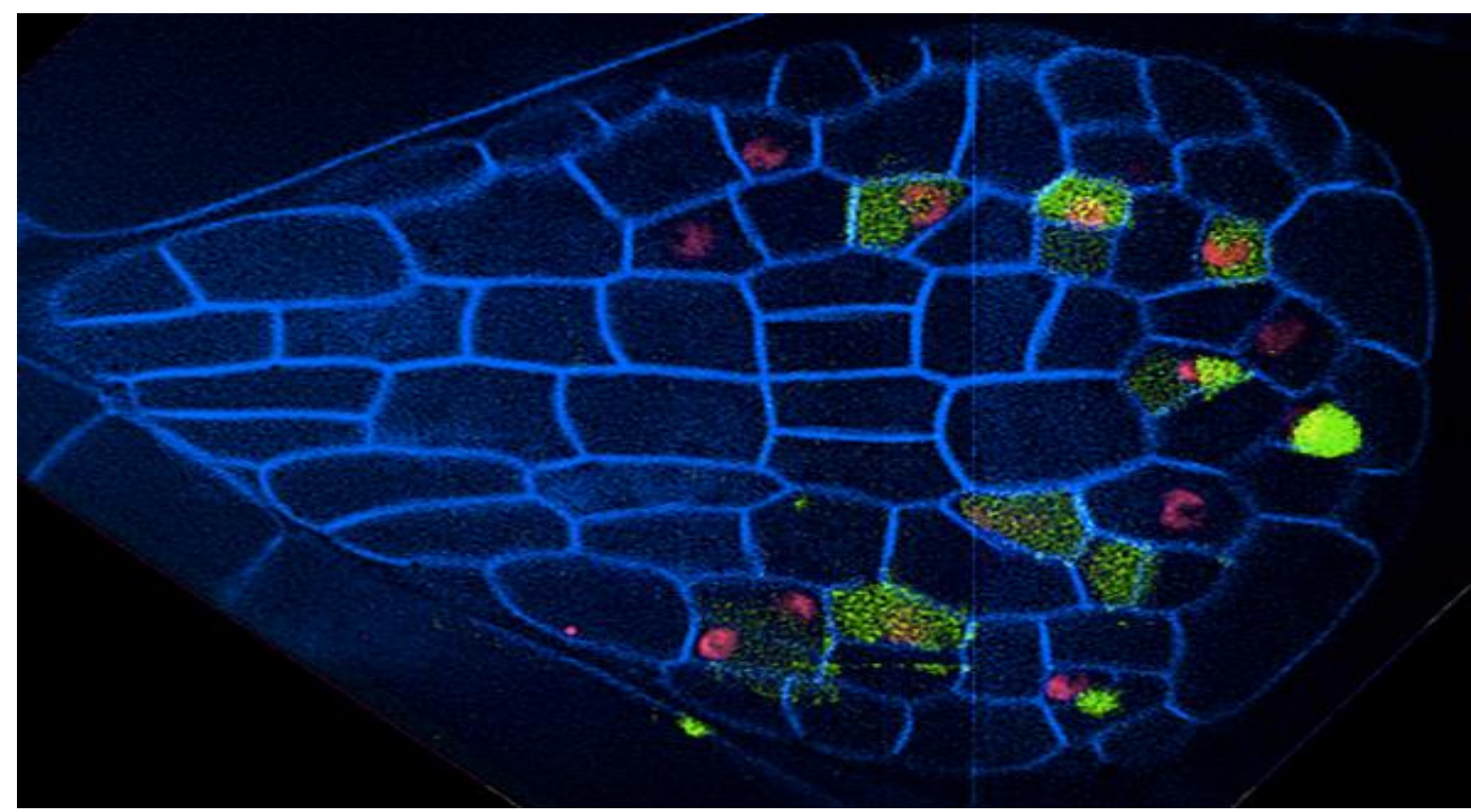

Figure 5 Input Microscopic Image

To getting images from the encompassing scene in the obvious bit of the electromagnetic spectrum (frequencies somewhere in the range of 400 and 700 nanometers). The light changes on the retina are shipped off image processor focus in the cortex. Assistant Maxwell appeared in the late nineteen century that each color image hack be made utilizing three images Red, green and blue image. A blend of these three images can create each color. This model, named RGB model, is principally utilized in image portrayal. The RGB image could be introduced as a triple(R, G, B) where normally R, G, and B take esteems in the reach [0, 255]. Another color model is YIQ model (overlay (Y), stage (I), quadrature stage (Q)). It is the base for the color TV standard. Images are introduced in PCs as a matrix of pixels. They have limited zone. On the off chance that we decline the pixel measurement the pixel splendor will turn out to be near the genuine brilliance.
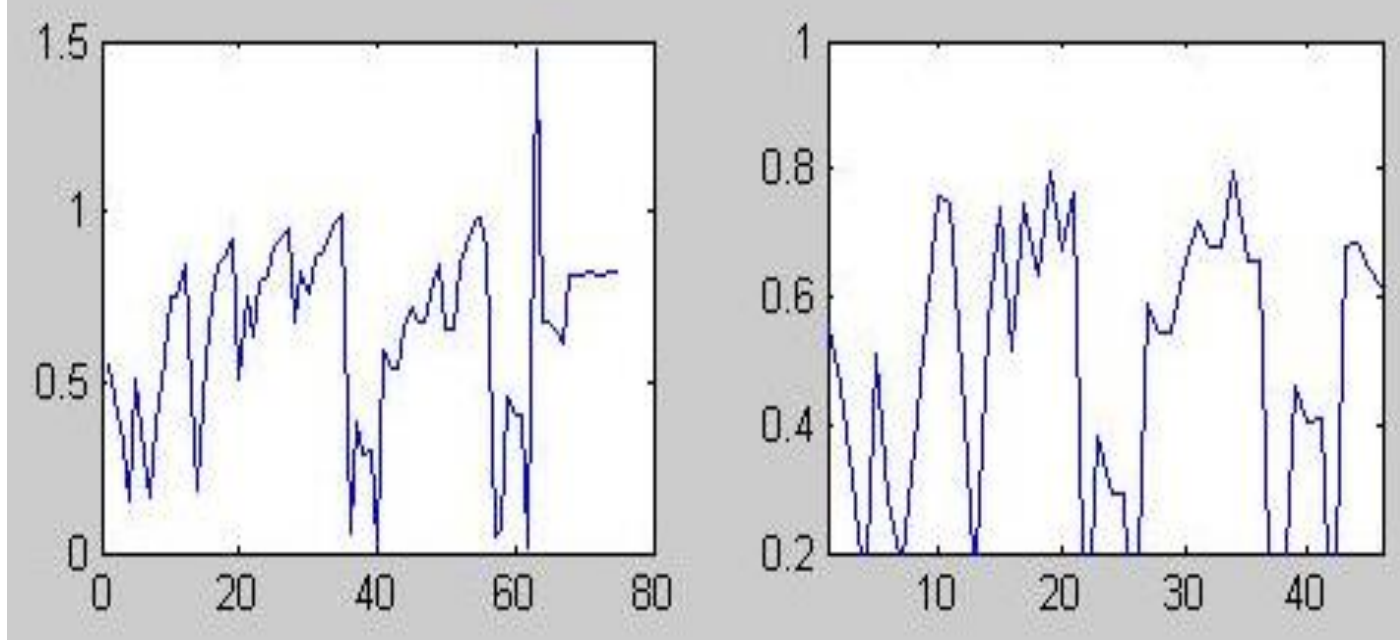

Figure 6Information Retrieval Process

\section{CONCLUSION}

In this paper we have actualized neural network based microscopic image retrieval for multi-image queries. Neural network is the development AI methods which has been utilizing for different pinnacles. In this paper neural network is utilized to learn, test and train the microscopic images and recovering required information from the images. Our approach bolsters any sort of microscopic images and consequently most significant information are recovered at absolute first layer of network training. Based on the proficient training of neural network the recovered information can additionally be prepared for exactness. In this paper, we portrayed the procedure and advancement of a neural network based image retrieval framework for microscopic images using a reference information base that contains images of more than one information. Consequently we have 
demonstrated that our proposed technique work better in recovering valuable information from any sort of microscopic images just as using multi image queries. This approach can be elaborated further to investigate microscopic image and the resultant image recovered from obscure images.

\section{REFERENCES}

1. Kashif, M., Raja, G. \& Shaukat, F. An Efficient Content-Based Image Retrieval System for the Diagnosis of Lung Diseases. J Digit Imaging 33, 971-987 (2020). https://doi.org/10.1007/s10278-020-00338-W.

2. Abeer Al-Mohamade 2020 "Multiple Query Content-Based Image Retrieval Using Relevance Feature Weight Learning" doi:10.3390/jimaging6010002

3. A.I. Shahin, Amira S. Ashour 2019 "Advanced neutrosophic sets in Microscopic Image Analysis"

4. Jiang, M.; Zhang, S.; Li, H.; Metaxas, D.N. Computer-Aided Diagnosis of Mammographic Masses Using Scalable Image Retrieval. IEEE Trans. Biomed. Eng. 2014, 62, 783-792. [CrossRef]

5. Zhang, X.; Liu, W.; Dundar, M.; Badve, S.; Zhang, S. Towards Large-Scale Histopathological Image Analysis: Hashing-Based Image Retrieval. IEEE Trans. Med. Imaging 2014, 34, 496-506.

6. Alraqibah, H.; Bchir, O.; Ismail, M.M.B. X-ray Image Retrieval System Based on Visual Feature Discrimination. In Proceedings of the International Conference on Digital Image Processing (ICDIP), Athens, Greece, 5-6 April 2014.

7. Komal, J.; Akhilesh, V.; Savita, G.; Swati, G. A Survey on Recent Image Indexing and Retrieval Techniques for Low-Level Feature Extraction in CBIR Systems. In Proceedings of the IEEE International Conference on Computational Intelligence \& Communication Technology (CICT), Ghaziabad, India, 13-14 February 2015.

8. Guo, J.M.; Prasetyo, H.; Chen, J.H. Content-Based Image Retrieval Using Error Diffusion Block Truncation Coding Features. IEEE Trans. Circ. Syst. Video Technol. 2014, 25, 466-481.

9. Thepade, S.D.; Shinde, Y.D. Robust CBIR using sectorisation of hybrid wavelet transforms with CosineWalsh, Cosine-Kekre, Cosine-Hartley combinations. In Proceedings of the International Conference on Pervasive Computing (ICPC), Pune, India, 8-10 January 2015. 